\title{
Farmacologia do fibratos
}

\author{
Pharmacology of fibrates
}

Hermes Toros Xavier

Divisão de Moléstias Cardiovasculares - Faculdade de Ciências Médicas de Santos - UNILUS

Os fibratos são as drogas de escolha nas hipertrigliceridemias. Após sua absorção, os fibratos são metabolizados pelo fígado, utilizando isoenzimas $\mathrm{P} 450$ não compartilhadas pelas estatinas. Entretanto, para alguns fibratos, como o genfibrozil, uma interação com estatinas pode ocorrer durante a sua glucuronidação, ou pelo deslocamento de frações livres de estatina ligadas às proteínas plasmáticas. A vida-média plasmática é variável entre os fibratos (2-80 h). Recentemente, foi demonstrado que os fibratos promovem suas ações lipídicas pelo estímulo dos PPAR$\alpha$. Através dessa ação, existe um incremento na transcrição de alguns genes relacionados com o metabolismo lipídico, como a LLP, APOAI, APOAll, ABCA-1, bem como uma diminuição na expressão da APOCIII, e muitas outras ações.

\section{Palavras-chave}

Fibratos, farmacologia, metabolismo lipídico
Fibrates are drugs commonly used in hypertriglyceridemias. After their absorption, fibrates are metabolized by the liver, using P450 isoenzymes not shared by statins. However, for some fibrates, such as genfibrozil, an interaction with statins can occur during their liver g/ucuronidation, or by displacement of free fraction of statins linked to proteins in plasma. Plasma half-life is variable among fibrates (2-80 h). Recently it has been shown that fibrates produce plasma lipid changes mainly by stimulating peroxisome-proliferation activation receptors alpha. Through this action, there is an increase in the transcription of some genes related to lipid metabolism, such as $L L P, A P O A l, A P O A l l, A B C A-1$, as well as decrease in the expression of APOCIII, and many other actions.

\section{KEY WORDS}

Fibrates, pharmacology, lipid metabolism

\section{INTRODUÇÃo}

Os fibratos ou derivados do ácido fíbrico são os medicamentos de escolha no tratamento da hipertrigliceridemia e têm um papel importante no controle das dislipidemias mistas. ${ }^{1}$ Estudos clínicos revelaram que os fibratos podem reduzir o risco de doença aterosclerótica coronariana (DAC) em pacientes com hipercolesterolemia e também, em indivíduos no pós infarto do miocárdio com níveis de LDL-colesterol (LDL-c) pouco elevados, de HDL-colesterol (HDL-c) baixos e com discretos aumentos de triglicérides (TG). ${ }^{2,3}$

Em estudos de regressão da aterosclerose avaliada pela angiografia, a terapia hipolipemiante com fibratos, diminuiu a progressão da aterosclerose e o aparecimento de novas lesões coronárias, em dois estudos e, em outro, mostrou uma redução significativa (40\%) na evolução das lesões coronárias focais em pacientes diabéticos. ${ }^{4-6}$

\section{Propriedades farmacológicas}

Os fibratos são absorvidos pelo trato gastrintestinal, glucuronidados pelo fígado e excretados pelos rins. 0 pico de concentração máxima dos fibratos pode variar entre 2 e $8 \mathrm{~h}$, a meia vida de eliminação entre 2 e $80 \mathrm{~h}$, dependendo das doses terapêuticas usadas, dos diferentes tipos de fibratos.

Devemos observar a possibilidade de interação medicamentosa dos fibratos com outros fármacos. As doses também devem ser adequadas em pacientes com disfunção renal ou hepática e nos idosos. Os fibratos não são metabolisados pelo citocromo P450 CYP 3A4, porém sua associação com estatinas deve ser feita com cuidado, pois pode levar a rabdomiólise. Isso ocorre devido à concentração desses medicamentos nos músculos. Na associação da cerivastatina com a genfibrosila, ocorreu um aumento da incidência de rabdomiólise e miosite, resultando na retirada da cerivastatina do mercado.

Os efeitos colaterais mais preocupantes com o uso dos fibratos são a elevação das aminotransferases (ALT, AST) e da creatinoquinase (CPK), o que ocorre em cerca de $0,1 \%$ dos pacientes. Da mesma forma que as estatinas para a ALT e AST são tolerados valores de até três vezes o normal e para o CPK de até dez vezes o valor normal. ${ }^{1,7}$

\section{MeCANISMo de aÇão e efEItos}

Os fibratos agem a partir da estimulação dos receptores nucleares ativados de proliferação dos peroxissomas-alfa, os conhecidos PPAR- $\alpha$, levando ao aumento da produção e da ação da lípase lipoprotéica (LPL) e redução da apoproteína CIII (Apo CIII), mecanismos estes, que estimulam a lipólise dos TG das VLDL-colesterol (VLDLc) e dos quilomícrons. Atuam também por diminuir a síntese das VLDL, pois reduzem a produção dos TG devido ao estímulo da beta oxidação dos ácidos graxos no fígado. Elevam os níveis de HDL-c pelo estímulo do PPAR- $\alpha$, 
que gera uma maior produção da $\mathrm{Apo} \mathrm{Al}$, e pelo incremento da lipólise de TG que também aumenta a oferta dos componentes de superfície das HDL. Dentre os principais efeitos dos fibratos, destacam-se, também, o aumento da excreção biliar do colesterol hepático e a redução do fluxo de ácidos graxos para o fígado.

Apresentam, ainda, ações antioxidantes, diminuindo a oxidação das LDL, ações antitrombóticas e antiinflamatórias, estas últimas, decorrentes também da ativação dos PPAR- $\alpha$, que inibe a expressão das interleucinas (IL1 e IL-6), da cicloxigenase-2 (COX-2), da proteína-C reativa (PCR), das moléculas de adesão, do fator tecidual, do fibrinogênio e do inibidor do ativador do plasminogênio (PAI-1). Em alguns ensaios clínicos, os fibratos diminuíram a resistência insulínica nos pacientes diabéticos. ${ }^{8,9}$

\section{Conclusão}

Pela sua significativa capacidade de reduzir TG, os fibratos se apresentam como uma das principais opções para o tratamento dos pacientes com hipertrigliceridemia, com ou sem colesterol elevado, incluindo os pacientes diabéticos tipo 2 com dislipidemia. Em média, os fibratos podem reduzir TG em $35 \%$, podendo chegar aos $60 \%$ nos pacientes hipertrigliceridêmicos graves; reduzir LDL-c de 10 a 31\%; diminuir a proporção de LDL-c pequena e densa (encontrada quando os níveis de TG estão acima de $150 \mathrm{mg} / \mathrm{dL}$ ); e elevar o HDL-c em até a $22 \% .{ }^{9}$

\section{REFERÊNCIAS}

1. Santos RD et al. III Diretrizes brasileiras sobre dislipidemias e diretriz de prevenção da aterosclerose do departamento de aterosclerose da sociedade brasileira de cardiologia. Arq Bras Cardiol. 2001;77( supl III):1-48.

2. Frick $\mathrm{MH}$ et al. Helsinki Heart Study: primary-prevention trial with gemfibrozil in middle-aged men with dyslipidemia.Safety of treatment, changes in risk factors, and incidence of coronary heart disease. N Engl J Med. 1987:317:1237-45

3. Rubins HB, Robins SJ. Conclusions from the VA-HIT study. Am J Cardiol.2000; 86:543-4.

4. Treatment Effects on Serum Lipoprotein Lipids, Apolipoproteins and Low Density Lipoprotein Particle Size and Relationships of Lipoprotein Variables to Progression of Coronary Artery Disease in the Bezafibrate Coronary Atherosclerosis Intervention Trial (BECAIT). J Am Coll Cardiol 1998;32:1648-56.
5. Frick MH et al. Prevention of the angiographic progression of coronary and vein-graft atherosclerosis by gemfibrozil after coronary bypass surgery in men with low levels of HDL cholesterol. Lopid Coronary Angiography Trial (LOCAT) Study Group. Circulation. 1997 ;96:2137-43.

6. Vakkilainen J et al.; DAIS Group. Relationships between low-density lipoprotein particle size, plasma lipoproteins, and progression of coronary artery disease: the Diabetes Atherosclerosis Intervention Study (DAIS). Circulation. $2003 ; 107: 1733-7$.

7. Chapman MJ. Fibrates in 2003: therapeutic action in atherogenic dyslipidemia and future perspectives. Atherosclerosis 2003;171:1-13.

8. Berger J, Moller DE. The mechanisms of action of PPARs. Annu Rev Med 2002; 53:409-35.

9. Martinez LRC, Santos RD. Papel dos fibratos no tratamento das dislipidemias In: Xavier HT(ed.) Manual de dislipidemias e cardiometabolismo. São Paulo: BBS Editora, 2004. p.157-165. 